\title{
Structure Design and Dynamical Modelling of a Spherical Robot Driven by Omnidirectional Wheels
}

\author{
Yonghua Huang, Ganmin Zhu, Changsheng Wang and Hao Huang \\ School of Mechanical and Electrical Engineering, Guilin University of Electronic Technology, Guilin, China, 541004
}

\begin{abstract}
In this paper, our work focused on designing a novel spherical robot driven by omnidirectional wheels and developing an under-actuated dynamical model for the system. The robot consists of four parts: the running spherical shell (1P), the supporting platform (1P), the driving omnidirectional wheels (3P) and the bearing ball wheels (3P). Considering the noholonomic constrains aroused from the contacts of the shell with the omnidirectional wheels, and the shell with ground, we derived a dynamical model for the system by Chaplygin dynamics. The model illustrated that the robot was an under-actuated system of six DOF (degree of freedom) and three driving-torque inputs. finally, we performed an inverse dynamics simulations of S-curve trajectory to show the effectiveness of the model.
\end{abstract}

\section{Introduction}

Spherical robot is a kind of mobile mechanical system typically consisting of a rolling shell and an enveloped driving mechanism. Due to the unique structure, spherical robot has some promise use in the fields of entertainment, wild detection and military patrolling, etc.

Driving method (or actuating mechanism) is a critical problem in developing a spherical robot. In tradition, there are two means to drive a spherical robot.

The first one is the eccentric moment driving mean. In this mean, the actuating mechanism of the spherical robot drives the COG(center of gravity) of the system deviating from the vertical sphere center line, and hence the sphere shell could run on ground. For example, in [1], A.Halme proposed a spherical robot with a single wheel set on the bottom of the spherical shell. When the wheel rolls along the inner surface of the shell, he believed, the eccentric moment would break the system's balance, so the robot shell began running accordingly. In [2], Ranjan Mukhejee presented a spherical robot driven by four moving masses. Mukhejee gave the argument when the four masses move along their individual leadscrews on the sphere diameter, the COM(center of mass) of the robot system should be alternated and consequently the shell would begin to roll. There are similar structures being proposed in [3]-[4]. In [5], J Alves considered a four wheels vehicle to drive his spherical robot. Alves suggested that the running vehicle on the bottom of the shell could give birth of sufficient moment to drive the robot to run. In [6], Zhan exploited an omnidirectional steering wheel and incorporate with a longitudinal driving wheel to drive a spherical robot. Zhan addressed that the cooperating rotations of the two wheels could guarentee the shell to run in all direction. Similar to Zhan, in [7], Wang also discussed a spherical robot of two omnidirectional wheels on two orthogonally axis. In [8], Sun suggested a two DOF pendulum in his spherical robot. Sun argued that the pendulum could be driven to swing on all sides, thus the robot certainly might run in omnidirection. In [9], Zhao presented a spherical robot with two coaxial eccentric masses. Zhao believed when the two masses run with different acceleration and velocity, the robot should move all direction accordingly. The similar structure was also proposed in [10].

The second one is the angular momentum driving mean. In this mean, the spherical robot usually configures with a high speed rotating rotor inside the sphere shell. Because the external friction torque between the running shell and the ground is commonly small, with the principle of the angular momentum conservation, the sphere shell would rotate in the direction reverse to that of the running rotor. Furthermore, by changing the speed and direction of the rotating rotor, the omnidirectional motion of the spherical robot could be easily get. For instance, in [11], Toshiaki Otani developed a spherical robot driven by a three axis mechanical gyroscope. The robot's gyroscope was fixed on a gimbal inside the sphere, and when it rotated with high speed, he believed, there would be a torque that can drive the sphere shell. Likewise, in [12], V.Joshi proposed a spherical robot driven by double rotors which shared the similar principle with the robot in [11].

As mentioned above, there are two kinds of driving means having been used in the existing spherical robot. However, comparing to the angular momentum driving mean(the latter), the spherical robot that use the eccentric moment driving mean(the former) has exhibited a lot of 
advantages, for instance, more flexibility of the steering, lower speed of the driving mechanism etc,. Specifically, the use of the technique of omnidirectional wheels in the driving mechanism of a spherical robot, just like which in [9] and [10], further highlights this point. As a conclusion, developing a novel structure of the driving mechanism system by utilizing the omnidirectional wheels become an attracting area in the field of the spherical robot research.

This paper deals with an innovating spherical robot driven by three inner omnidirectional wheels. The paper is arranged as follow: at first, we show the mechanical structure of the proposed spherical robot in detail; and next, we develop a dynamical model for the robot system by using Chaplygin equation; and at the end, we consider a S-curve trajectories of the spherical robot system and perform an inverse dynamics simulations to validate the effectiveness of the proposed model.

\section{General scheme}

In [9] [10], the omnidirectional wheel were used in the spherical robots successfully. However, their design one wheel contacting with the shell, which might weaken the driving capability of the system, so we use three omnidirectional wheels to improve our system's performance.

\subsection{Mechanical structure}

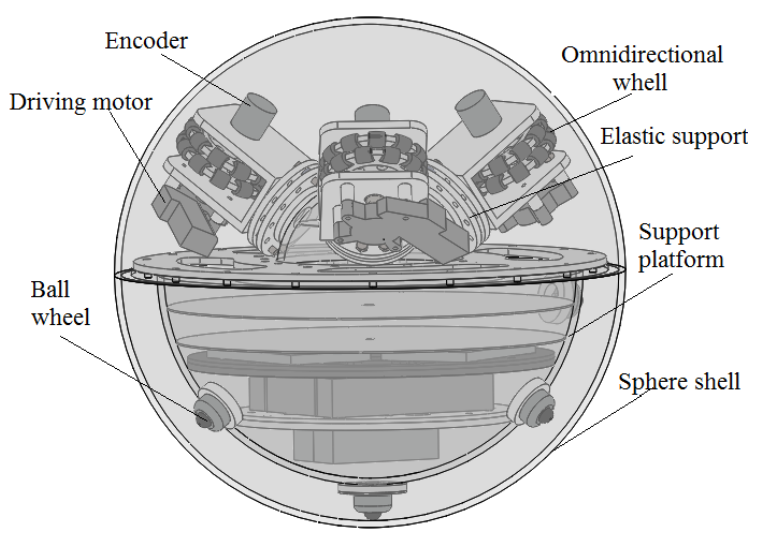

Figure 1. Virtual prototype of our spherical robot.

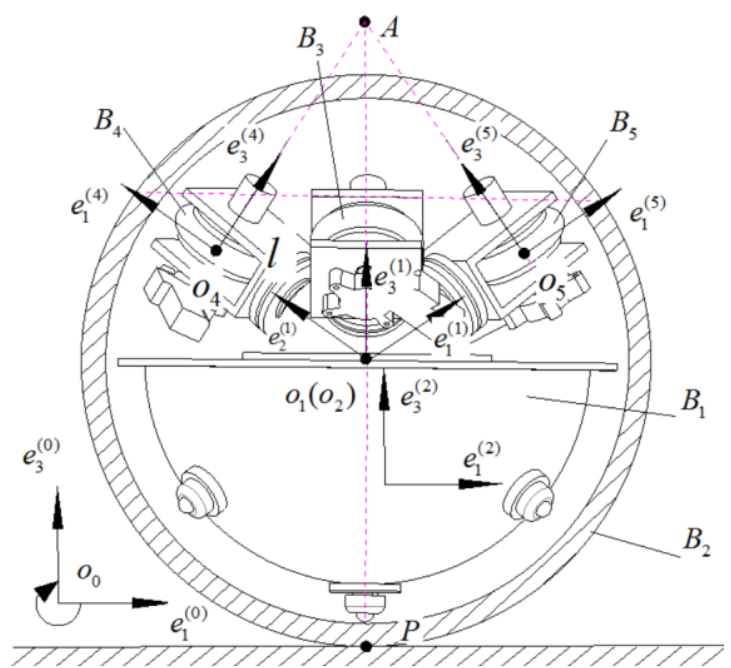

Figure 2. The schematic diagram of our spherical robot.
Our spherical robot approximately consists of two parts: a sphere shell and a support platform. The structure of the system is shown in Fig. 1 Fig. 2.

As it is seen in Fig 1 Fig 2, the three omnidirectional wheels are symmetrically assigned on the upper half part of the inner platform. Additionally, it is noteworthy that, for preventing the three wheels from leaving the internal surface of the sphere shell, we exploit an elastic support for each of the omnidirectional wheel.

Moreover, for ensuring the stability of our system, we exploit three free-running ball wheels on the bottom of the shell to bear the platform. Similarly, the ball wheels are also mounted on the platform via an elastic support.

As for the actuators, three thin motors are used to drive the three omnidirectional wheels to rotate relative to the inner sphere support platform.

\subsection{Working principle}

The working principle of the proposed spherical robot can be summarized as follow:

The motors drive the three omnidirectional wheels to run with a given law. Simultaneously, the running wheels would synthesize a couple to propel the shell to roll via the contacting friction. And hence, we can conclude if we regulate the rotation speed and direction of the wheels, we could drive the sphere shell in all direction.

After the omnidirectional wheels being driven, because the three wheels are all mounted on the inner support platform, there should be a reaction couple acting on the platform by the omnidirectional wheels. With the bearing action of the three free running ball wheels, the reaction couple would drive the support platform to rotate about the geometric center of the shell.

If we configure the COG of the support platform in different position (that can be done by putting auxiliary weights on the platform), the support platform could work either as a rotor of high speed rotating or a pendulum of low speed swinging.

\section{Dynamical model}

In this section, we will develop a dynamical model for the robot system by using Chaplygin equation.

\subsection{Coordination settings}

We represent the supporting platform as $B_{1}$, and the sphere shell as $B_{2}$, and the omnidirectional wheels as $B_{i} \quad(i=3,4,5)$. The coordinates of the robot are set as:

- $O-\boldsymbol{e}_{1} \boldsymbol{e}_{2} \boldsymbol{e}_{3}\{0\}$ is the global coordinate system fixed on the ground;

- $O_{1}-\boldsymbol{e}_{1}^{(1)} \boldsymbol{e}_{2}^{(1)} \boldsymbol{e}_{3}^{(1)}\{1\}$ is the coordinate system of the inner supporting platform $B_{1}$ and its origin is the geometric center of the sphere shell $B_{2}$;

- $O_{2}-\boldsymbol{e}_{1}^{(2)} \boldsymbol{e}_{2}^{(2)} \boldsymbol{e}_{3}^{(2)}\{2\}$ is the coordinate system of the shell $B_{2}$ and its origin is the same as $\{1\}$ 
$O_{i}-\boldsymbol{e}_{1}^{(i)} \boldsymbol{e}_{2}^{(i)} \boldsymbol{e}_{3}^{(i)}\{i\}(i=3,4,5)$ is the coordinate system of the omnidirectional wheel $B_{i}$ and the origin locates at its geometric center.

\subsection{Noholonomic constrains}

We suppose the robot runs on a horizontal plane, then the angular velocity of $B_{1}$ can be given as:

$$
\boldsymbol{\omega}_{B 1}^{(1)}=\left(c_{3} \dot{q}_{2}-c_{2} s_{3} \dot{q}_{1}\right) \boldsymbol{e}_{1}^{(1)}+\left(s_{2} \dot{q}_{1}+\dot{q}_{3}\right) \boldsymbol{e}_{2}^{(1)}+\left(c_{2} c_{3} \dot{q}_{1}+s_{3} \dot{q}_{2}\right) \boldsymbol{e}_{3}^{(1)}
$$

where $\boldsymbol{e}_{i}^{(j)}(i=1,2,3, j=1,2, \cdots)$ is the $i$ th base vector of coordinate $\{j\} \cdot s_{i}=\sin \left(q_{i}\right), c_{i}=\cos \left(q_{i}\right)(i=1,2, \cdots)$; $\dot{q}_{i}(i=1,2,3)$ is the $i$ th Eular angular rate of $B_{1}$.

Similarly, the angular velocity of $B_{2}$ can be given as:

$$
\boldsymbol{\omega}_{B 2}^{(2)}=\dot{q}_{5} \boldsymbol{e}_{1}^{(2)}+c_{5} \dot{q}_{6} \boldsymbol{e}_{2}^{(2)}+\left(\dot{q}_{4}+s_{5} \dot{q}_{6}\right) \boldsymbol{e}_{3}^{(2)}
$$

where $\dot{q}_{i}(i=4,5,6)$ is the $(i-3)$ th angular rate of $B_{2}$.

Because $B_{i}(i=3,4,5)$ rotates about $B_{1}$, their angular velocity should be calculated as:

$$
\boldsymbol{\omega}_{B j}^{(j)}={ }^{j} \boldsymbol{R}_{1} \bullet \boldsymbol{\omega}_{B 1}^{(1)}+\dot{q}_{(j+4)} \boldsymbol{e}_{3}^{(j)} \quad(j=3,4,5)
$$

where ${ }^{j} \boldsymbol{R}_{i}(i, j=1,2, \cdots)$ denotes the rotation transform matric from the coordinate $\{i\}$ to $\{j\}$, and $\dot{q}_{i}(i=7,8,9)$ denotes the angular rate of $B_{i}(i=3,4,5)$, respectively.

We assume the robot runs on a plane without slipping, so the velocity of the point $P$ is straightforward satisfied:

$$
\boldsymbol{v}_{o 2}^{(0)}+\left({ }^{0} \boldsymbol{R}_{2} \cdot \boldsymbol{\omega}_{B 2}^{(2)}\right) \times \boldsymbol{R}^{(0)}=0
$$

where $\boldsymbol{R}^{(0)}$ is the position vector of $P$ in coordinate $\{0\}$.

If we assume $\boldsymbol{v}_{o 2}^{(0)}$ have the form: $\boldsymbol{v}_{o 2}^{(0)} \triangleq \dot{\boldsymbol{x}} \boldsymbol{e}_{1}^{(0)}+\dot{y} \boldsymbol{e}_{2}{ }^{(0)}$ and considered the first two items in Equ. (4), we would get two noholonomic constrains as:

$$
\left\{\begin{array}{l}
\dot{q}_{5}=\frac{s_{4} \dot{x}-c_{4} \dot{y}}{l+r} \\
\dot{q}_{6}=\frac{c_{4} \dot{x}+s_{4} \dot{y}}{(l+r) c_{5}}
\end{array}\right.
$$

where $l$ is the distance between the geometer center of $B_{2}$ and the geometer center of $B_{i}(i=3,4,5)$.

Similar to the presumption of that the shell running on ground, here we also assume $B_{i}(i=3,4,5)$ all run on the inner surface of $B_{1}$ without slipping, then we will get the other noholonomic constrains:

$$
\begin{array}{r}
\dot{q}_{i}=g_{(6 i-5)} \dot{x}+g_{(6 i-4)} \dot{y}+g_{(6 i-3)} \dot{q}_{4}+\sum_{j=3}^{5} g_{(6 i-5+j)} \dot{q}_{(4+j)} \\
(i=1,2,3)(9) \sim(11)
\end{array}
$$

In (7) (9), $\dot{x}, \dot{y}$ denote the longitudinal and the lateral velocity of the geometric center of $B_{2}$, respectively; $g_{j}(j=1,2,3, \cdots, 18)$ is the function of $q_{i}(i=1,2,3,4,5)$.

\subsection{Velocities of the COM}

We set the velocity of the geometric center of $B_{2}$ as:

$$
\boldsymbol{v}_{C 2}^{(0)}=\dot{x} \boldsymbol{e}_{1}^{(0)}+\dot{y} \boldsymbol{e}_{2}^{(0)}
$$

Under the principle of the relative motion, we will get respectively the velocity of $B_{i}(i, j=1,2, \cdots, 5)$ as:

$$
\begin{gathered}
\boldsymbol{v}_{C 1}^{(1)}={ }^{1} \boldsymbol{R}_{0} \cdot \boldsymbol{\bullet}_{o 2}^{(0)}+\boldsymbol{\omega}_{B 1}^{(1)} \times \boldsymbol{l}_{C 1}^{(1)} \\
\boldsymbol{v}_{C 2}^{(0)}=\dot{x} \boldsymbol{e}_{1}^{(0)}+\dot{y} \boldsymbol{e}_{2}^{(0)} \\
\boldsymbol{v}_{C k}^{(1)}={ }^{1} \boldsymbol{R}_{0} \cdot \boldsymbol{v}_{o 2}^{(0)}+\boldsymbol{\omega}_{B 1}^{(1)} \times\left(\boldsymbol{l}_{o k}^{(1)}+{ }^{1} \boldsymbol{R}_{i} \cdot \boldsymbol{l}_{C i}^{(k)}\right)(k=3,4,5)(15) \sim
\end{gathered}
$$

where $\boldsymbol{l}_{C i}^{(i)}(i=1,3,4,5)$ is the position vector in coordinate $\{i\}$ from the center of $B_{2}$ to that of $B_{i} ; \boldsymbol{l}_{o i}^{(1)} \quad(i=3,4,5)$ is the position vector in $\{1\}$ from the geometric center of $B_{2}$ to the geometric center of $B_{i}(i=3,4,5)$.

\subsection{Kinetic energy}

According to the derived $\boldsymbol{\omega}_{B i}^{(j)} \quad(i, j=1,2, \cdots, 5)$ in (1) (5) and $\boldsymbol{v}_{C i}^{(k)}(i=1,2, \cdots, 5 ; k=0,1)$ in $(10) \sim(14)$, we can calculate the system's kinetic energy as:

$$
T=\frac{1}{2} \sum_{i=1}^{5}\left(\left(\boldsymbol{\omega}_{B i}^{(j)}\right)^{T} \boldsymbol{J}_{B i}\left(\boldsymbol{\omega}_{B i}^{(j)}\right)+\left(\boldsymbol{v}_{C i}^{(k)}\right)^{T} M_{B i}\left(\boldsymbol{v}_{C i}^{(k)}\right)\right)
$$

where $\boldsymbol{J}_{B i}\left(\boldsymbol{M}_{i}\right)(i, j=1,2, \cdots, 5)$ represents the inertial matric (mass matrix) of $B_{i}(i, j=1,2, \cdots, 5)$, respectively.

By substituting the noholonomic constrains (5) (9) into $T$, we will get another form of the kinetic energy $\tilde{T}$.

\subsection{Generalized forces}

The external force acting on our spherical robot consist of the driving torque of $B_{i}(i=3,4,5)$ and the gravity.

We consider the COM of $B_{1}$ and $B_{i}(i=3,4,5)$ in the coordinate $\{1\}$, which is represented as:

$$
\boldsymbol{l}_{C}^{(1)}=\frac{1}{m_{1}+\sum_{i=3}^{5} m_{i}}\left(m_{1} \boldsymbol{l}_{C 1}^{(1)}+\sum_{i=3}^{5} m_{i}\left(\boldsymbol{l}_{o i}^{(1)}+{ }^{1} \boldsymbol{R}_{i} \boldsymbol{l}_{C i}^{(i)}\right)\right)
$$

If we defined $h \triangleq{ }^{1} \boldsymbol{R}_{i} \cdot \boldsymbol{l}_{C}^{(1)}[3]$ (where (*)[3] is the $3 \mathrm{rd}$ item of the vector*), we will get $h$ is the function of $q_{i}(i=2,3)$. 
Moreover, if we investigate the first time derivative of $h$, then we will get:

$$
\dot{h}=f_{1} \dot{q}_{2}+f_{2} \dot{q}_{3}
$$

In (18), $f_{i}(i=1,2)$ denotes the explicit function of $q_{i} \quad(i=2,3)$ and $m_{j}(j=1,3,4,5)$.

With (18), we define $\tau_{g} \triangleq\left(\begin{array}{lll}0 & \tau_{g 2} & \tau_{g 3}\end{array}\right)^{T}$, in which

$$
\tau_{g k}=\left(m_{1}+\sum_{i=3}^{5} m_{i}\right) g \bullet f_{(k-1)} \quad k=2,3
$$

Again, according to (7) (9), we define a transform matric as follow:

$$
\boldsymbol{R}_{\tau} \triangleq\left(\begin{array}{llllll}
g_{1} & g_{2} & g_{3} & g_{4} & g_{5} & g_{6} \\
g_{7} & g_{8} & g_{9} & g_{10} & g_{11} & g_{12} \\
g_{13} & g_{14} & g_{15} & g_{16} & g_{17} & g_{18}
\end{array}\right)^{T}
$$

Consequently, with (19)and(20), the generalized forces of the system can be calculated as

$$
\tilde{\boldsymbol{Q}}=\boldsymbol{Q}+\boldsymbol{R}_{\tau} \cdot \boldsymbol{\tau}_{g}
$$

where $\boldsymbol{Q}=\left(\begin{array}{llllll}0 & 0 & 0 & \tau_{7} & \tau_{8} & \tau_{9}\end{array}\right)^{T}, \tau_{i}(i=7,8,9)$ is the driving torque of $B_{j}(j=3,4,5)$.

\subsection{Dynamical model}

Considering the following form of Chaplygin equation

$$
\frac{d}{d t} \frac{\partial \tilde{T}}{\partial \dot{q}_{\sigma}}-\frac{\partial \tilde{T}}{\partial q_{\sigma}}-\sum_{\beta=1}^{\gamma} \frac{\partial T}{\partial \dot{q}_{\varepsilon+\beta}} \sum_{v=1}^{\varepsilon}\left(\frac{\partial B_{\varepsilon+\beta, \sigma}}{\partial q_{v}}-\frac{\partial B_{\varepsilon+\beta, v}}{\partial q_{\sigma}}\right) \dot{q}_{v}=\tilde{Q}_{\sigma}
$$

where $T$ is the kinetic energy and $\tilde{T}$ is the kinetic energy by substituting noholonomic constrains into $T$; $B_{\varepsilon+\beta, \sigma}$ is the $\sigma$ th coefficient of the $\beta$ th noholonomic constrain; $q_{v}$ and $q_{\sigma}$ are the generalized coordinates of the system; $\varepsilon$ and $\gamma$ are the numbers of the independent generalized coordinates and the noholonomic constrains; $\tilde{Q}_{\sigma}$ is the $\sigma$ th generalized force of the system.

We can get the system's dynamics as

$$
\boldsymbol{D}(\boldsymbol{q}) \ddot{\boldsymbol{q}}_{\theta}+\boldsymbol{C}\left(\boldsymbol{q}, \dot{\boldsymbol{q}}_{\theta}\right) \dot{\boldsymbol{q}}_{\theta}+\boldsymbol{G}(\boldsymbol{q})=\boldsymbol{Q}
$$

In $(23), \quad \boldsymbol{D}(\boldsymbol{q}) \in \mathfrak{R}^{6 \times 6}, \boldsymbol{C}\left(\boldsymbol{q}, \dot{\boldsymbol{q}}_{\theta}\right) \in \mathfrak{R}^{6 \times 6} \quad, \quad$ and $\boldsymbol{G}(\boldsymbol{q}) \in \mathfrak{R}^{6 \times 1}$ denote the inertia, centripetal-Coriolis, and gravity terms; $\boldsymbol{q}_{\theta}$ and $\boldsymbol{q}$ are two kinds of generalized coordinates, which are defined as:

$$
\boldsymbol{q}_{\theta}=\left(\begin{array}{llllll}
x & y & q_{4} & q_{7} & q_{8} & q_{9}
\end{array}\right)^{T},
$$

$$
\boldsymbol{q}=\left(\begin{array}{lllll}
q_{1} & q_{2} & q_{3} & q_{4} & q_{5}
\end{array}\right)^{T} .
$$

Equation (23) indicates the spherical robot is explicitly an under-actuated system with six independent velocities, and for more detail:

The longitudinal displacementand lateral displacement $(x, y)$ of the geometric center of $B_{1}$, and the yaw angle $\left(q_{4}\right)$ of $B_{1}$ are all under-actuated;

There are totally three driving inputs in the three joints $q_{i}(i=7,8,9)$, therefore we could regulate control-force inputs $\tau_{i}(i=7,8,9)$ of the three joints to control the trajectory $\left(x, y, q_{4}\right)$ of the sphere shell.

\section{Inverse dynamics simulations}

In order to prove the reliability of the proposed model (see Equ. (25)), here we consider the derived dynamical

\begin{tabular}{|c|c|c|c|c|}
\hline Physical meanings & Sym & \multicolumn{3}{|c|}{ value } \\
\hline $\begin{array}{c}\text { Radius of the } \\
\text { omnidirectional wheel (m) }\end{array}$ & $r$ & \multicolumn{3}{|c|}{0.06} \\
\hline $\begin{array}{l}\text { Distance between the } \\
\text { geometer center of the shell } \\
\text { and the omnidirectional } \\
\text { wheel }(\mathrm{m})\end{array}$ & $l$ & \multicolumn{3}{|c|}{0.25} \\
\hline $\begin{array}{l}\text { Distance between the } \\
\text { geometer center of the shell } \\
\text { and the COG of the } \\
\text { supporting platform }(\mathrm{m})\end{array}$ & $L_{1}$ & \multicolumn{3}{|c|}{0.20} \\
\hline $\begin{array}{c}\text { Distance between geometer } \\
\text { center of the } \\
\text { omnidirectional wheel and } \\
\text { the its COG }(\mathrm{m})\end{array}$ & $L_{2}$ & \multicolumn{3}{|c|}{0.25} \\
\hline $\begin{array}{l}\text { Mass of the supporting } \\
\text { platform (kg) }\end{array}$ & $m_{1}$ & \multicolumn{3}{|c|}{17.57} \\
\hline $\begin{array}{c}\text { Mass of the sphere shell } \\
(\mathrm{kg})\end{array}$ & $m_{2}$ & \multicolumn{3}{|c|}{2.87} \\
\hline $\begin{array}{l}\text { Mass of the omnidirectional } \\
\text { wheel }(\mathrm{kg})\end{array}$ & $m_{3}$ & \multicolumn{3}{|c|}{0.45} \\
\hline \multirow{3}{*}{$\begin{array}{l}\text { Inertia matrix of the } \\
\text { supporting platform } \\
\qquad\left(\mathrm{kg} \cdot \mathrm{m}^{2}\right)\end{array}$} & \multirow{3}{*}{$J_{B 1}$} & $(0.78$ & 0 & 0 \\
\hline & & 0 & 0.78 & 0 \\
\hline & & 0 & 0 & 0.78 \\
\hline \multirow{3}{*}{$\begin{array}{l}\text { Inertia matrix of the sphere } \\
\text { shell }\left(\mathrm{kg} \cdot \mathrm{m}^{2}\right)\end{array}$} & \multirow{3}{*}{$J_{B 2}$} & 0.13 & 0 & 0 \\
\hline & & 0 & 0.13 & 0 \\
\hline & & 0 & 0 & 0.13 \\
\hline \multirow{3}{*}{$\begin{array}{l}\text { Inertia matrix of the } \\
\text { omnidirectional wheel } \\
\qquad\left(\mathrm{kg} \cdot \mathrm{m}^{2}\right)\end{array}$} & \multirow{3}{*}{$J_{B 3}$} & $(0.001$ & 0 & 0 \\
\hline & & 0 & 0.001 & 0 \\
\hline & & 0 & 0 & 0.002 \\
\hline
\end{tabular}
model, an perform inverse dynamics of S-curve trajectory for the spherical robot.

Table 1 shows the physical parameters which would be used in the numerical simulation. Note that we obtain the parameters from the measurement of a virtual bicycle prototype in Solidworks.

Table 1. Physical parameters.

The simulation is perform with three steps.

- Step 1: trajectory planning 
We plan the motion trajectory of the geometric center of the sphere shell $B_{2}$ :

$x(t)=A_{1} t ; \dot{x}(t)=A_{1} ; \quad \ddot{x}(t)=0 ;$

$y(t)=A_{2} e^{-\sigma t} \sin (\omega t+\varphi) ;$

$\dot{y}(t)=A_{2} e^{-\sigma t}(\omega \cos (\omega t+\varphi)-\sigma \sin (\omega t+\varphi)) ;$

$\ddot{y}(t)=A_{2} e^{-\sigma t}\left(\left(\sigma^{2}-\omega^{2}\right) \sin (\omega t+\varphi)-2 \sigma \omega \cos (\omega t+\varphi)\right)$

$q_{4}(t)=0 ; \quad \dot{q}_{4}(t)=0 ; \quad \ddot{q}_{4}(t)=0 ;$

where $A_{1}=0.2, A_{2}=0.1, \sigma=0.01, \omega=0.4 \pi, \varphi=0$.

\section{Step 2: motion state calculating}

We investigate the first three items of the dynamical model (Equ.(23))

$$
\boldsymbol{D}_{u 1}(\boldsymbol{q}) \ddot{\boldsymbol{q}}_{\theta u}+\boldsymbol{D}_{a 1}(\boldsymbol{q}) \ddot{\boldsymbol{q}}_{\theta a}+\boldsymbol{F}_{1}\left(\boldsymbol{q}, \dot{\boldsymbol{q}}_{\theta}\right)=\boldsymbol{0}
$$

where $\ddot{\boldsymbol{q}}_{\theta u}=\left(\begin{array}{lll}\ddot{x} & \ddot{y} & \ddot{q}_{4}\end{array}\right)^{T}, \ddot{\boldsymbol{q}}_{\theta a}=\left(\begin{array}{lll}\ddot{q}_{7} & \ddot{q}_{8} & \ddot{q}_{9}\end{array}\right)^{T}$, and $\boldsymbol{F}_{1}\left(\boldsymbol{q}, \dot{\boldsymbol{q}}_{\theta}\right) \in \mathfrak{R}^{3 \times 1}$ denotes the first three items of the vector $\left(\boldsymbol{C}\left(\boldsymbol{q}, \dot{\boldsymbol{q}}_{\theta}\right) \dot{\boldsymbol{q}}_{\theta}+\boldsymbol{G}(\boldsymbol{q})\right)$, and

$$
\boldsymbol{D}_{u 1}(\boldsymbol{q})=\left(\begin{array}{ccc}
D_{11} & D_{12} & D_{13} \\
D_{21} & D_{22} & D_{23} \\
D_{31} & D_{32} & D_{33}
\end{array}\right), \boldsymbol{D}_{a 1}(\boldsymbol{q})=\left(\begin{array}{ccc}
D_{14} & D_{15} & D_{16} \\
D_{24} & D_{25} & D_{26} \\
D_{34} & D_{35} & D_{36}
\end{array}\right),
$$

and $D_{i j}(i, j=1,2, \cdots, 6)$ is the corresponding component of $\boldsymbol{D}(\boldsymbol{q})$. Then we will get

$$
\ddot{\boldsymbol{q}}_{\theta a}=-\left(\boldsymbol{D}_{u}(\boldsymbol{q})\right)^{-1} \boldsymbol{F}_{1}\left(\boldsymbol{q}, \dot{\boldsymbol{q}}_{\theta}\right)
$$

From (25), we can calculate angular rotational rate $\dot{\boldsymbol{q}}_{\theta a}$ by the numerical time integration.

Subsequently, with the derived $\dot{\boldsymbol{q}}_{\theta a}$, we further examine the (5) (9), then we get $\dot{q}_{i}(i=1,2,3,5,6)$.

Similarly, by integration of $\dot{q}_{i}(i=1,2,3,5,6)$, we will obtain $q_{i}$, definitely.

\section{- Step 3: driving torque calculating}

Similar to Step 2, we furtherly investigate the remain three items of the dynamical model(Equ.(23)), and then we will obtain:

$$
\boldsymbol{\tau}_{a}=\boldsymbol{D}_{u 2}(\boldsymbol{q}) \ddot{\boldsymbol{q}}_{\theta u}+\boldsymbol{D}_{a 2}(\boldsymbol{q}) \ddot{\boldsymbol{q}}_{\theta a}+\boldsymbol{F}_{2}\left(\boldsymbol{q}, \dot{\boldsymbol{q}}_{\theta}\right)
$$

where $\boldsymbol{\tau}_{a}=\left(\begin{array}{lll}\tau_{7} & \tau_{8} & \tau_{9}\end{array}\right)^{T}, \quad \boldsymbol{F}_{2}\left(\boldsymbol{q}, \dot{\boldsymbol{q}}_{\theta}\right) \in \mathfrak{R}^{3 \times 1}$ denotes the later three items of the vector $\left(\boldsymbol{C}\left(\boldsymbol{q}, \dot{\boldsymbol{q}}_{\theta}\right) \dot{\boldsymbol{q}}_{\theta}+\boldsymbol{G}(\boldsymbol{q})\right)$, and

$\boldsymbol{D}_{u 2}(\boldsymbol{q})=\left(\begin{array}{ccc}D_{41} & D_{42} & D_{43} \\ D_{51} & D_{52} & D_{53} \\ D_{61} & D_{62} & D_{63}\end{array}\right), \boldsymbol{D}_{a 2}(\boldsymbol{q})=\left(\begin{array}{ccc}D_{44} & D_{45} & D_{46} \\ D_{54} & D_{55} & D_{56} \\ D_{64} & D_{65} & D_{66}\end{array}\right)$.

Figure 3 shows the desired trajectory of the inverse dynamics simulation.

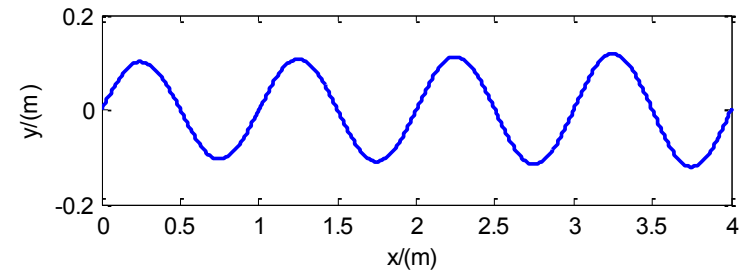

Figure 3. The planning trajectory of the COM of the shell.

Figure 4 demonstrates the driving angular velocity and angular acceleration of the omnidirectional wheels.

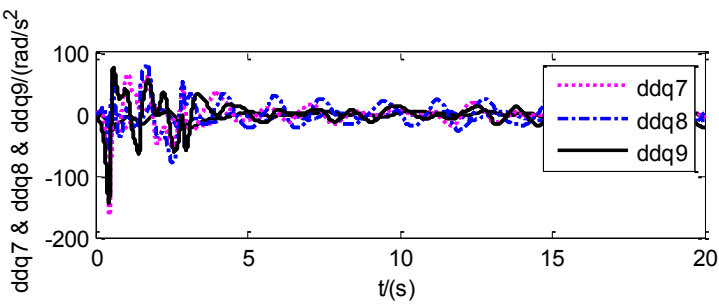

a) Angular acceleration

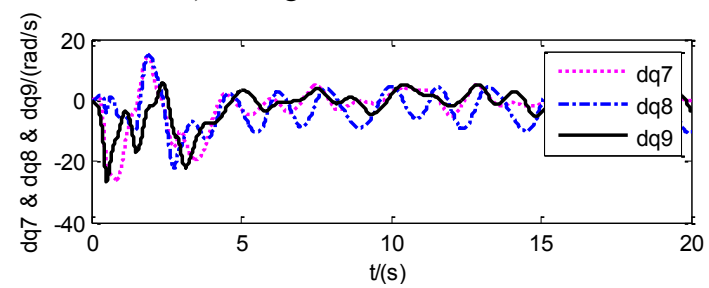

b) Angular velocity

Figure 4. The state of the omnidirectional wheel

Figure 5 shows the velocity constrains of the support platform and the sphere shell.

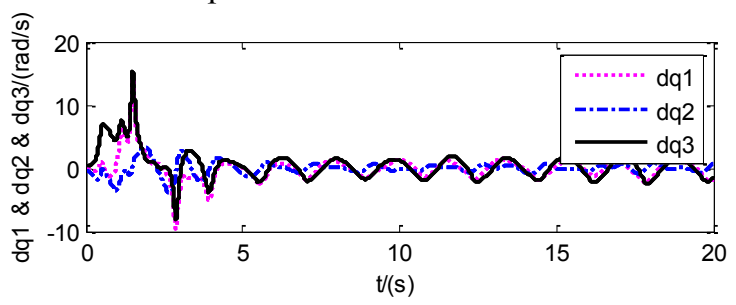

a) Angular rate of the platform

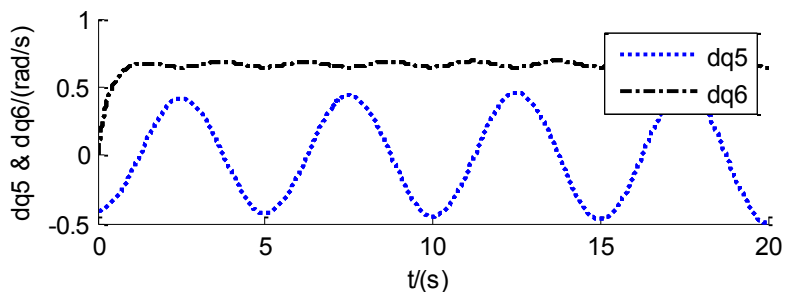

b) Angular rate of the shell

Figure 5. The noholonomic constrains.

Figure 6 shows the driving torques and the powers of the three omnidirectional wheels.

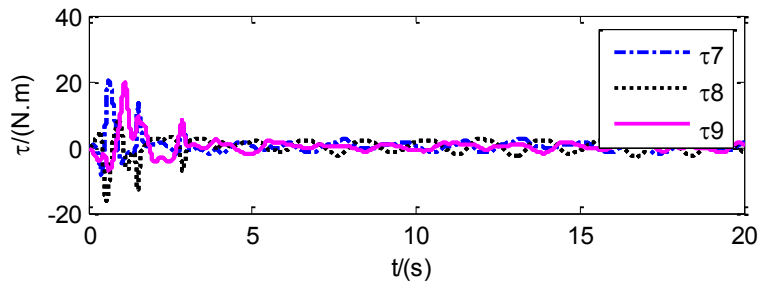

a) Input torques of the omnidirectional wheels 


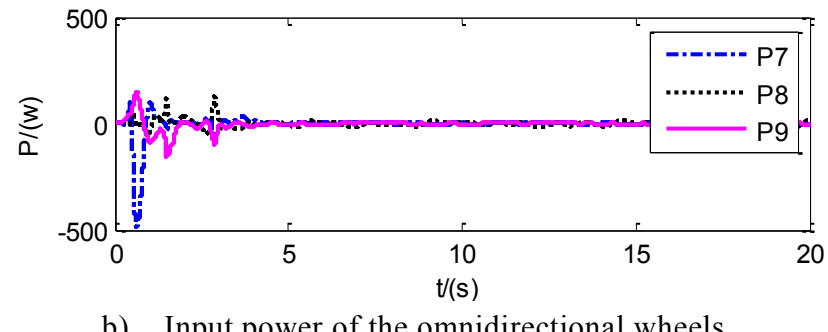

b) Input power of the omnidirectional wheels

Figure 6. The torques and the powers of the omnidirectional wheel.

As a conclusion, the results show that the maximum driving torque of the three omnidirectional wheels is less than 20N.m(see Fig 6(a)), and the maximum input power is less than 500W(see Fig 6(b)). The results are useful in the selection of the driving motor.

Moreover, considering the results in Fig 3 Fig 6, we investigate the comparison between the kinetic energy of the system and the work of the external forces.

Figure 7 examines two kinds of kinetic energy: T1 is get by the current velocity and $\mathrm{T} 2$ is by the previous velocity and the elementary work.

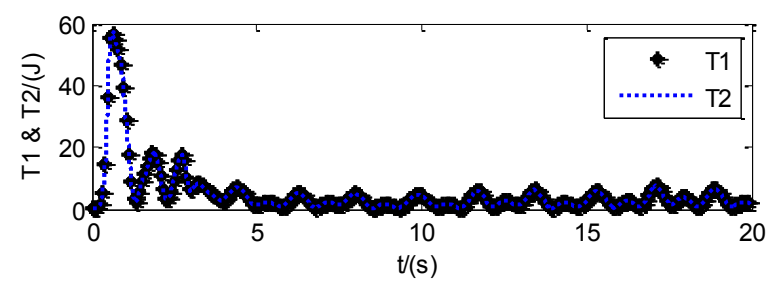

Figure 7. The comparison of two kinds of kinetic energy.

Figure 8 shows the difference between the increment of the kinetic energy and the elementary work.

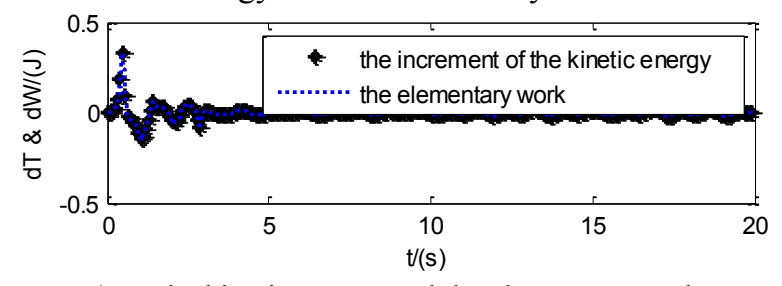

a) The kinetic energy and the elementary work

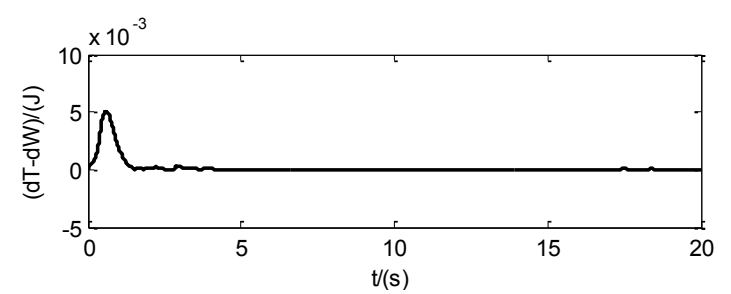

b) The difference of the increment of the kinetic energy and the elementary work

Figure 8. The kinetic energy Vs the elementary work.

As seen in Fig 7 Fig 8, while calculated by use of different variable, the two kinetic energy are coincident, and concretely, their difference is less than $10^{-2}$ (see Fig $8(\mathrm{a})$ ). The results show the fact that our dynamical model (Equ. (23)) completely obey the law of conservation of energy, thus to a certain extent, we verify reliability of our derivation.

\section{Conclusions and future work}

As a summary, one of the contribution of this research is that we proposed a novel spherical robot that can be driven by three omnidirectional wheels inside a sphere shell. Another contribution of the research might be that we explored the dynamical model for the system. Our derivation illustrates that the spherical robot is explicitly a noholonomic and under-actuated system, which consist of six independent velocities and three control-torque inputs. With the inverse dynamics simulations of an S-curve trajectory, we validated that the feasibility of our structure scheme of the robot system and the reliability of our dynamical model.

However, currently in our research, there definitely is lack of physical porotype experiments to provide further support for our theoretical analysis, therefore our next work should concentrate on the development a principle prototype so as to perform the presented motion trajectory of the robot system.

\section{Acknowledgement}

This work was financial supported by National Natural Science Foundation of China (Grant No.61365012 \&\& No.51305087\&\&No.51765011) and Director Foundation of Guangxi Key Laboratory of Manufacturing Systems and Advanced Manufacturing Technology (Grant No. 14045-15- 003Z).

\section{References}

1. Halme A, Schonberg T, Wang Y. Motion control of a spherical mobile robot[C]. In Proceedings of the 4th International Workshop on Advanced Motion Control. 1996: 259-264.

2. Mukherjee R, Minor M A. A simple motion planner for a spherical mobile robot[C]. Proceedings of the 1999 IEEE International Conference on Advanced Intelligent Mechatronics. 1999: 896-901.

3. Amir H J A, Mojabi P. Introducing Glory: A novel strategy for an omnidirectional spherical rolling robot[J]. Journal of Dynamic Systems Measurement \& Control, 2004, 126(3): 678-683.

4. LI Tuan-jie, SU Li, ZHANG Yan. Design and Analysis of a Spherical Omnidirectional Rolling Robot Driven by Linear Motors[J]. Machine Design and Research, 2006, 22(4): 46-48.

5. Alves J, Dias J. Design and control of a spherical mobile robot[J]. Proceedings of the Institution of Mechanical Engineers Part I Journal of Systems \& Control Engineering, 2003, 217(6): 457-467.

6. Zhan Q, Cai Y, Yan C. Design, analysis and experiments of an omin-directional spherical robot [C]. IEEE International Conference on Robotics and Autmation. 2011: 4921-4926.

7. Wang Kejun. Mechanism Analysis and Research of Spherical Robot Driven by Omnidirectional Wheel [D]. Beijing Jiaotong University, 2017.

8. SUN Hanxu, WANG Liangqing, JIA Qingxuan, LIU Daliang. Dynamic Model of the BYQ-3 Spherical 
Robot [J]. Journal of Mechanical Engineering, 2009, 45 (10): 8-14.

9. ZHAO Bo, LI Man-tian, SUN Li-nin. Turning in place motion control of two pendulums driven spherical robot[J]. Journal of Harbin Institute of Technology, 2011, 43(11): 49-53.

10. Yoon J C, Ahn S S, Lee Y J. Spherical robot with new type of two-pendulum driving mechanics[C]. IEEE International Conference on Intelligent Engineering Systems. 2011: 275-279.
11. Otani T, Urakubo T, Maekawa S, et el. Positional and attitude control of a spherical rolling robot equipped with a gyro[C]. IEEE International Workshop on Advance Motion Control. 2006: 416-421.

12. Joshi V A, Banavar R N, Hippalgaonkar R. Design and analysis of a spherical mobile robot[J]. Mechanism \& Machine Theory, 2010, 45(5): 130136. 